\title{
Iterative Algorithms for a System of Generalized Mixed Equilibrium Problems ${ }^{1}$
}

\author{
Yali Zhao \\ Bohai University \\ College of Mathematics and Physics \\ Jinzhou, liaoning China, 121013 \\ Yalizhao2000@163.com
}

\begin{abstract}
The purpose of this paper is to present an iterative algorithm for a system of generalized mixed equilibrium problems(for short, denoted by SGMEP) in Banach space. We prove strong convergence theorems of the iterative algorithm for finding a common element of the fixed point set of relatively nonexpansive mappings and the solution set of the SGMEP in Banach space under some suitable conditions.

Keywords-System of generalized mixed equilibrium problems; Iterative algorithm; Relatively nonexpansive mappings; Strong convergence
\end{abstract}

\section{I.INTRODUCTION}

The equilibrium problem, which was first introduced by Blum and Oettli[1], provides a unified model of many problems such as optimization problems, variational inequality problems, complementarity problems, fixed point problems and so on. And the equilibrium problem is the special case of the generalized mixed equilibrium problem. The generalized mixed equilibrium problem plays an important role in economic, management and engineering. Iterative methods for nonexpansive mappings have recently been applied with generalized mixed equilibrium problem. In this paper, we introduce an SGMEP and an iterative algorithm for the SGMEP is suggested for finding a common element of the fixed point set of relatively nonexpansive mappings and the solution set of the SGMEP in Banach space under some suitable conditions. The results obtained here extend and improve the corresponding results of [2-4].

In this paper, we consider the SGMEP of finding $x \in C$ such that

$$
\left\{\begin{array}{c}
F_{1}(x, y)+\left\langle\eta(y, x), A_{1} x\right\rangle \geq 0, \forall y \in C, \\
F_{2}(x, y)+\left\langle\eta(y, x), A_{2} x\right\rangle \geq 0, \forall y \in C, \\
\vdots \\
F_{N}(x, y)+\left\langle\eta(y, x), A_{N} x\right\rangle \geq 0, \forall y \in C,
\end{array}\right.
$$

where $F_{i}(x, y)=f_{i}(x, y)+\phi(x, y)-\phi(x, x), i=1,2, \cdots, N$. The solution set to the SGMEP is denoted by $\operatorname{SGMEP}\left(F_{1}, F_{2}, \cdots, F_{N}\right)$.

This work is supported by the National Natural Science Foundation of China (11371070).

\author{
Dianjiao Zhao \\ Bohai University \\ College of Mathematics and Physics \\ Jinzhou, Liaoning China, 121013 \\ 1033718547@qq.com
}

\section{II.PRELIMINARIES}

Let $E$ be a Banach space whose norm is denoted by $\|\cdot\|$. Let $C$ be a nonempty closed subset of $E$. Let $J$ be the normalized duality mapping defined by

$$
J x=\left\{x^{*} \in E^{*}:\left\langle x, x^{*}\right\rangle=\|x\|\left\|x^{*}\right\|,\|x\|=\left\|x^{*}\right\|\right\}, x \in E,
$$

where $E^{*}$ denotes the dual space of $E$ and $\langle\cdot$,$\rangle is the$ generalized duality pairing between $E$ and $E^{*}$. It is also known that if $E$ is uniformly smooth, then $J$ is uniformly norm-tonorm continuous on each bounded subset of $E$. Let $A: C \rightarrow E^{*}$ be nonlinear mapping. Let $F: C \times C \rightarrow R$ be a bifunction. We know the following: If $E$ is smooth, then $J$ is single-valued; If $E$ is reflexive, then $J$ is surjective;If $E$ is uniformly convex, then it is reflexive; If $E$ is a reflexive and strictly convex, then $J^{-1}$ is norm-weak-continuous; $E$ is uniformly smooth if and only if $E^{*}$ is uniformly convex; If $E^{*}$ is uniformly convex, then $J$ is uniformly norm-to-norm continuous on each bounded subset of $E$. If $E$ is a smooth, strictly convex and reflexive Banach space and $C$ is a nonempty closed convex subset of $E$. we denote by $\phi$ the function defined by $\phi(x, y)=\|x\|^{2}-2\langle x, J y\rangle+\|y\|^{2}, \forall x, y \in E$.

Following Alber [8], the generalized projection $\prod_{C}: E \rightarrow C$ is a map that assigns to an arbitrary point $x \in E$ the minimum point of the functional $\phi(x, y)$, that is, $\prod_{C} x=\bar{x}$, where $\bar{x}$ is the solution to the minimization problem $\phi(\bar{x}, x)=\inf _{y \in C} \phi(y, x)$.

Existence and uniqueness of the operator $\prod_{C}$ follows from the properties of the functional $\phi(x, y)$ and strict monotonicity of the mapping $J$. It is obvious from the definition of function $\phi$ that

$$
(\|y\|-\|x\|)^{2} \leq \phi(y, x) \leq(\|y\|+\|x\|)^{2}, \forall x, y \in E .
$$

Define a function $V: E \times E^{*} \rightarrow R$ as follows

$$
V\left(x, x^{*}\right)=\|x\|^{2}-2\left\langle x, x^{*}\right\rangle+\left\|x^{*}\right\|^{2}, \forall\left(x, x^{*}\right) \in E \times E^{*} .
$$

Then, it is obvious that $V\left(x, x^{*}\right)=\phi\left(x, J^{-1}\left(x^{*}\right)\right)$ and $V(x, J(y))=\phi(x, y)$. If $E$ is a Hilbert space, then $\phi(x, y)=\|x-y\|^{2}$. If $E$ is a reflexive, strictly convex and 
smooth Banach space, then for all $x, y \in E, \phi(x, y)=0$ if and only if $x=y$. It is sufficient to show that if $\phi(x, y)=0$, then $x=y$, thus $\|x\|=\|y\|$. This implies that

$\langle x, J y\rangle=\|x\|^{2}=\|J y\|^{2}$. From the definition of $J$, one has $J x=J y$. Therefore, we have $x=y$ (see [2, 3] for more details). In order to obtain our main results, we need the following definitions and lemmas.

Definition 2.1 Let $\eta: C \times C \rightarrow E$, and $A: C \rightarrow E^{*}$ be mappings, $A$ is $\eta$-monotone mapping if

$$
\langle A x-A y, \eta(x-y)\rangle \geq 0, \forall x, y \in C \text {. }
$$

Definition $2.2^{[2]}$ A point $x \in C$ is a fixed point of a mapping $T: C \rightarrow C$ if $T x=x$. The set of fixed points of $T$ is denoted by $T_{\text {Fix }}$, that is, $T_{F i x}=\{x \in C \mid T x=x\}$.

Definition $2.3^{[2]}$ A point $p$ in $C$ is said to be an asymptotic fixed point of $T$ if $C$ contains a sequence $\left\{x_{n}\right\}$ which converges weakly to $p$ such that $\lim _{n \rightarrow \infty}\left\|x_{n}-T x_{n}\right\|=0$, the set of asympotic fixed points of $T$ is denoted by $\hat{T}_{F i x}$.

Definition $2.4^{[2]}$ A mapping $T$ from $C$ into itself is said to be relatively nonexpansive if $\hat{T}_{\text {Fix }}=T_{\text {Fix }}$ and $\varphi(p, T x) \leq \varphi(p, x)$ for all $x \in C$ and $P \in T_{F i x}$.

Lemma $2.1^{[6]}$ Let $E$ be a reflexive, strictly convex, and smooth Banach space, let $C$ be a nonempty closed convex subset of $E$ and let $x \in E$. Then

$$
\phi\left(y, \Pi_{C} x\right)+\phi\left(\Pi_{C} x, x\right) \leq \phi(y, x), \forall y \in E .
$$

Lemma 2.2 Define $T_{r}: E \rightarrow C$ as follows:

$$
\begin{aligned}
& T_{r}(x)=\{z \in C, F(z, y)+\langle\eta(y, z), A z\rangle+ \\
& \left.\frac{1}{r}\langle y-z, J z-J x\rangle \geq 0 . \forall y \in C\right\}
\end{aligned}
$$

Then for all $(p, x) \in\left(T_{r}\right)_{\text {Fix }} \times E$,

$$
\phi\left(p, T_{r} x\right)+\phi\left(T_{r} x, x\right) \leq \phi(p, x) .
$$

where $\left(T_{r}\right)_{\text {Fix }}$ denotes the fixed point sets of $T_{r}$.

Lemma $2.3^{[6]}$ Let $C$ be a nonempty closed convex subset of a smooth Banach space $E$, and $x \in E$. Then, $x_{0}=\prod_{C} x$ if and only if

$$
\left\langle x_{0}-y, J x-J x_{0}\right\rangle \geq 0, \forall y \in C .
$$

Lemma $2.4^{[7]}$ Let $E$ be a strictly convex and smooth real Banach space and let $\left\{x_{n}\right\}$ and $\left\{y_{n}\right\}$ be two sequences of
$E$. If $\phi\left(x_{n}, y_{n}\right) \rightarrow 0$, and either $\left\{x_{n}\right\}$ or $\left\{y_{n}\right\}$ is bounded. Then $\left\|x_{n}-y_{n}\right\| \rightarrow 0$. For solving SGMEP, let $F: C \times C \rightarrow R$ be a bifunction, $\eta: C \times C \rightarrow E$ be a mapping and $\phi: C \rightarrow R$ be a convex and lower semicontinuous function satisfying the following conditions:

(A1) $F(x, x)=0$, for all $x \in C$; (A2) $F$ is monotone, i.e., $F(x, y)+F(y, x) \leq 0$,for all $x, y \in C$; (A3) For each $x, y, z \in C, \limsup F(t z+(1-t) x, y) \leq F(x, y)$;

(A4) For each $x \in C, y \mapsto F(x, y)$ is convex and lower semicontinuous; (A5) $\eta(x, y)=-\eta(y, x), \forall x, y \in C$;

(A6) For $y \in C, x \mapsto \eta(x, y)$ is continuous nonlinear mapping; (A7) For $x \in C, y \mapsto \eta(x, y)$ is continuous mapping.

\section{MAIN RESULTS}

Theorem3.1. Let $E$ be uniformly convex and uniformly smooth Banach space, let $C$ be a nonempty closed convex subset of $E$. Let $F_{i}: C \times C \rightarrow R$ and $\eta: C \times C \rightarrow E$ satisfy (A1) - (A7) with $i=1,2, \cdots, N$. Let $A_{i}: C \rightarrow E^{*}$ $(i=1,2, \cdots, N)$ be continuous and $\eta$ - monotone mapping, $J$ be uniformly norm-to-norm continuous on bounded set. And let $T, S$ be a relatively nonexpansive mapping from $C$ into itself such that $\Gamma=S_{F i x} \cap T_{F i x} \cap \operatorname{SGMEP}\left(F_{1}, F_{2}, \cdots F_{N}\right) \neq \varnothing$. Let $\left\{x_{n}\right\}$ be a sequence generated by $x_{1}=x \in C$ and $C_{0}=C$,

$$
\begin{gathered}
u_{n}^{1} \in C, F_{1}\left(u_{n}^{1}, y\right)+\left\langle\eta\left(y, u_{n}^{1}\right), A_{1} u_{n}^{1}\right\rangle+\frac{1}{r_{n}^{1}}\left\langle y-u_{n}^{1},\right. \\
\left.J u_{n}^{1}-J x_{n}\right\rangle \geq 0, \forall y \in C, \\
u_{n}^{2} \in C, F_{2}\left(u_{n}^{2}, y\right)+\left\langle\eta\left(y, u_{n}^{2}\right), A_{2} u_{n}^{2}\right\rangle \\
+\frac{1}{r_{n}^{2}}\left\langle y-u_{n}^{2}, J u_{n}^{2}-J u_{n}^{1}\right\rangle \geq 0, \forall y \in C, \\
\vdots \\
u_{n}^{N} \in C, F_{N}\left(u_{n}^{N}, y\right)+\left\langle\eta\left(y, u_{n}^{N}\right), A_{N} u_{n}^{N}\right\rangle+ \\
\frac{1}{r_{n}^{N}}\left\langle y-u_{n}^{N}, J u_{n}^{N}-J u_{n}^{N-1}\right\rangle \geq 0, \forall y \in C, \\
z_{n}=\prod_{C} J^{-1}\left(\gamma_{n} J u_{n}^{N}+\left(1-\gamma_{n}\right) J T u_{n}^{N}\right), \\
w_{n}=J^{-1}\left(\beta_{n} J x_{n}+\left(1-\beta_{n}\right) J S z_{n}\right), \\
C_{n+1}=\left\{z \in C_{n}: \phi\left(z, w_{n}\right) \leq \beta_{n} \phi\left(z, x_{n}\right)+\right. \\
\left.\left(1-\beta_{n}\right) \phi\left(z, z_{n}\right) \leq \phi\left(z, x_{n}\right)\right\}, \\
x_{n+1}=\prod_{C_{n+1}} x_{0}, \forall n \geq 0, x_{0} \in C .
\end{gathered}
$$


for $n \in N$, where $\Pi_{C}$ is the generalized projection from $E$ onto $C$. The coefficient sequences $\left\{\gamma_{n}\right\},\left\{\beta_{n}\right\} \subset[0,1]$ satisfying $\lim \sup \beta_{n}<1$. If $T, S$ are uniformly continuous, then the sequence $\left\{x_{n}\right\}$ converges strongly to $\Pi_{\Gamma} x_{0}$.

Proof. we first show that $\left\{x_{n}\right\}$ is bounded. Let

$$
p \in \Gamma=S_{F i x} \cap T_{F i x} \cap \operatorname{SGMEP}\left(F_{1}, F_{2}, \cdots F_{N}\right) .
$$

From Lemma 1.2, we can have $\phi\left(p, u_{n}^{1}\right) \leq \phi\left(p, x_{n}\right)$.

So we get

$\phi\left(p, u_{n}^{N}\right) \leq \phi\left(p, u_{n}^{N-1}\right) \leq \cdots \leq \phi\left(p, u_{n}^{1}\right) \leq \phi\left(p, x_{n}\right) ，$

Which implying that

$$
\begin{aligned}
\phi\left(p, z_{n}\right) & =\phi\left(p, \prod_{C} J^{-1}\left(\gamma_{n} J u_{n}^{N}+\left(1-\gamma_{n}\right) J T u_{n}^{N}\right)\right) \\
& \leq \phi\left(p, J^{-1}\left(\gamma_{n} J u_{n}^{N}+\left(1-\gamma_{n}\right) J T u_{n}^{N}\right)\right) \\
& \leq \phi\left(p, u_{n}^{N}\right) \leq \phi\left(p, x_{n}\right) .
\end{aligned}
$$

By the definition of the function $V$ and the latter inequality, weget

$$
\phi\left(p, w_{n}\right)=\phi\left(p, J^{-1}\left(\beta_{n} J x_{n}+\left(1-\beta_{n}\right) J S z_{n}\right)\right) \leq \phi\left(p, x_{n}\right)
$$

Hence, for $\forall n \geq 0$, we get $p \in C_{n+1}$, and also $\left\{x_{n}\right\}$ is well defined. From Lemma 2.3 and $x_{n}=\prod_{C_{n}} x_{0}$, we have

$$
\begin{aligned}
& \left\langle x_{n}-z, J X_{0}-J x_{n}\right\rangle \geq 0, \forall z \in C_{n} . \\
& \left\langle X_{n}-p, J x_{0}-J x_{n}\right\rangle \geq 0, \forall p \in C_{n} .
\end{aligned}
$$

From Lemma 2.1, one has

$$
\begin{aligned}
& \phi\left(x_{n}, x_{0}\right)=\phi\left(\prod_{C} x_{0}, x_{0}\right) \leq \phi\left(p, x_{0}\right)-\phi\left(p, x_{n}\right) \\
& \leq \phi\left(p, x_{0}\right)
\end{aligned}
$$

For all $p \in \Gamma \in C_{n}$ and $n \geq 1$. then, the sequence $\left\{\phi\left(x_{n}, x_{0}\right)\right\}$ is bounded. Since $\left(\left\|x_{n}\right\|-\left\|x_{0}\right\|\right)^{2} \leq \phi\left(x_{n}, x_{0}\right)$. It implies that $\left\{x_{n}\right\}$ is bounded. Also know $\left\{z_{n}\right\},\left\{w_{n}\right\}$ $\left\{u_{n}^{1}\right\}, \cdots,\left\{u_{n}^{N}\right\}$ are all bounded. Since $x_{n}=\prod_{C_{n}} x_{0}$ and $x_{n+1}=\prod_{C_{n+1}} x_{0} \in C_{n+1} \subset C_{n}$, we have

$$
\phi\left(x_{n}, x_{0}\right) \leq \phi\left(x_{n+1}, x_{0}\right), \forall n \in N \text {. }
$$

Therefore $\left\{\phi\left(x_{n}, x_{0}\right)\right\}$ is nondecreasing. Hence, the limit of $\left\{\phi\left(x_{n}, x_{0}\right)\right\}$ exists. By the construction of $C_{n}$, one has that
$C_{m} \subset C_{n}$ and $x_{m}=\prod_{C_{m}} x_{0} \in C_{n}$ for any positive integer $m \geq n$. It follows that

$$
\begin{aligned}
\phi\left(x_{m}, x_{n}\right) & =\phi\left(x_{m}, \prod_{C_{n}} x_{0}\right) \\
& \leq \phi\left(x_{m}, x_{0}\right)-\phi\left(\prod_{C_{n}} x_{0}, x_{0}\right) \\
& =\phi\left(x_{m}, x_{0}\right)-\phi\left(x_{n}, x_{0}\right)
\end{aligned}
$$

Letting $m, n \rightarrow \infty$ in the latter inequalities, we get $\phi\left(x_{m}, x_{n}\right) \rightarrow 0$. It follows from Lemma 2.4, that $\left\|x_{m}-x_{n}\right\| \rightarrow 0$ as $m, n \rightarrow \infty$. That is , $\left\{x_{n}\right\}$ is a Cauchy sequence. There exists a sequence $\left\{x_{n_{i}}\right\}$ of $\left\{x_{n}\right\}$ such that $\lim _{n \rightarrow \infty} x_{n}=u \in C$. Next we show $u \in T_{F i x} \cap S_{F i x}$. By the construction of $C_{n}$, we know $\phi\left(z, w_{n}\right) \leq \beta_{n} \phi\left(z, x_{n}\right)+\left(1-\beta_{n}\right) \phi\left(z, z_{n}\right) \leq \phi\left(z, x_{n}\right)$.

So $\left(1-\beta_{n}\right) \phi\left(z, z_{n}\right) \leq\left(1-\beta_{n}\right) \phi\left(z, x_{n}\right)$. For $\beta_{n} \in(0,1)$, one has $\phi\left(z, z_{n}\right) \leq \phi\left(z, x_{n}\right)$. For $x_{n+1}=\prod_{C_{n+1}} x_{0} \in C_{n+1}$, we have $\phi\left(x_{n+1}, z_{n}\right) \leq \phi\left(x_{n+1}, x_{n}\right)$. From $\lim _{n \rightarrow \infty} \phi\left(x_{n+1}, x_{n}\right)=0$, we get $\lim _{n \rightarrow \infty} \phi\left(x_{n+1}, z_{n}\right)=0$.From Lemma 2.4, we have $\lim _{n \rightarrow \infty}\left\|x_{n+1}-z_{n}\right\|=0$. Since $J$ is uniformly norm-to-norm continuous on bounded set, noting that

$$
\lim _{n \rightarrow \infty}\left\|J x_{n+1}-J z_{n}\right\|=0 \text {. }
$$

For

$$
\begin{aligned}
& \phi\left(p, z_{n}\right)-\phi\left(p, x_{n}\right) \\
\leq & \left\|z_{n}-x_{n}\right\|\left(\left\|z_{n}\right\|+\left\|x_{n}\right\|\right)+2\|p\| \cdot\left\|J z_{n}-J x_{n}\right\| \cdot
\end{aligned}
$$

It follows from the latter inequality, we obtain that $\lim _{n \rightarrow \infty}\left(\phi\left(p, z_{n}\right)-\phi\left(p, x_{n}\right)\right)=0$. And thus we have

$$
\phi\left(p, z_{n}\right) \leq \phi\left(p, u_{n}^{N}\right) \leq \phi\left(p, x_{n}\right) .
$$

So $\lim _{n \rightarrow \infty} \phi\left(p, z_{n}\right)=\lim _{n \rightarrow \infty} \phi\left(p, u_{n}^{N}\right)=\lim _{n \rightarrow \infty} \phi\left(p, x_{n}\right)$. And

$$
\begin{aligned}
& \phi\left(p, u_{n}^{N}\right)-\phi\left(p, x_{n}\right) \\
= & \left\|u_{n}^{N}\right\|^{2}-\left\|x_{n}\right\|^{2}-2\left\langle p, J u_{n}^{N}-J x_{n}\right\rangle \\
\leq & \left\|u_{n}^{N}\right\|^{2}-\left\|x_{n}\right\|^{2}+2\|p\| \cdot\left\|J u_{n}^{N}-J x_{n}\right\| \\
\leq & \left\|u_{n}^{N}-x_{n}\right\| \cdot\left(\left\|u_{n}^{N}\right\|+\left\|x_{n}\right\|\right)+2\|p\| \cdot\left\|J u_{n}^{N}-J x_{n}\right\| .
\end{aligned}
$$

Thus $\quad \lim _{n \rightarrow \infty}\left\|u_{n}^{N}\right\|=\lim _{n \rightarrow \infty}\left\|x_{n}\right\|$. Implying that $\lim _{n \rightarrow \infty}\left\|x_{n}\right\|=\lim _{n \rightarrow \infty}\left\|z_{n}\right\|=\lim _{n \rightarrow \infty}\left\|u_{n}^{N}\right\|=u$. Furthermore, we get 
$\phi\left(p, z_{n}\right) \leq \gamma_{n} \phi\left(p, u_{n}^{N}\right)+\left(1-\gamma_{n}\right) \phi\left(p, T u_{n}^{N}\right) \leq \phi\left(p, u_{n}^{N}\right)$

From $\left\{\gamma_{n}\right\} \subset[0,1]$ and as $n \rightarrow \infty$, noting

$\lim _{n \rightarrow \infty} \phi\left(p, z_{n}\right) \leq \lim _{n \rightarrow \infty} \gamma_{n} \phi\left(p, u_{n}^{N}\right)+\lim _{n \rightarrow \infty}\left(1-\gamma_{n}\right) \phi\left(p, T u_{n}^{N}\right)$

$\leq \lim _{n \rightarrow \infty} \phi\left(p, u_{n}^{N}\right)$.

Which means that $\lim _{n \rightarrow \infty} \phi\left(p, u_{n}^{N}\right)=\lim _{n \rightarrow \infty} \phi\left(p, T u_{n}^{N}\right)$. Thus

$$
\begin{aligned}
& \phi\left(p, u_{n}^{N}\right)-\phi\left(p, T u_{n}^{N}\right) \\
& \leq\left\|u_{n}^{N}-T u_{n}^{N}\right\| \cdot\left(\left\|u_{n}^{N}\right\|+\left\|T u_{n}^{N}\right\|\right)+2\|p\| \cdot\left\|J u_{n}^{N}-J T u_{n}^{N}\right\| .
\end{aligned}
$$

From above inequality and $J$ is uniformly norm-to-norm continuous on bounded set, we get

$$
\lim _{n \rightarrow \infty}\left\|u_{n}^{N}-T u_{n}^{N}\right\|=0, \lim _{n \rightarrow \infty}\left\|J u_{n}^{N}-J T u_{n}^{N}\right\|=0 .
$$

By the definition of $T$ and $\left\|u_{n}^{N}\right\| \rightarrow\|u\|$, as $n \rightarrow \infty$, we have $T u=u$, which implies that $u \in T_{\text {Fix }}$. From the definition of $\phi$ and definition 2.4, we have

$$
\lim _{n \rightarrow \infty} \phi\left(x_{n}, w_{n}\right) \leq \lim _{n \rightarrow \infty}\left(1-\beta_{n}\right) \phi\left(x_{n}, z_{n}\right)=0 .
$$

So $\lim _{n \rightarrow \infty}\left\|x_{n}-w_{n}\right\|=0$. And

$$
\left\|J X_{n+1}-J w_{n}\right\| \geq\left(1-\beta_{n}\right)|| J X_{n+1}-J S z_{n}\left\|-\beta_{n}\right\| J X_{n}-J X_{n+1} \| .
$$

Hence $\left\|J X_{n+1}-J S z_{n}\right\| \leq \frac{1}{1-\beta_{n}}\left(\left\|J X_{n+1}-J w_{n}\right\|+\beta_{n}\left\|J X_{n}-J X_{n+1}\right\|\right) \cdot \quad$ It follows from $\limsup _{n \rightarrow \infty} \beta_{n}<1$ that $\lim _{n \rightarrow \infty}\left\|J x_{n+1}-J S z_{n}\right\|=0$.

Since $J$ is uniformly norm-to-norm continuous on bounded set, we have $\lim _{n \rightarrow \infty}\left\|x_{n+1}-S z_{n}\right\|=0$. Note that

$$
\left\|z_{n}-S z_{n}\right\| \leq\left\|z_{n}-x_{n+1}\right\|+\left\|x_{n+1}-S z_{n}\right\| \text {, So } \lim _{n \rightarrow \infty}\left\|z_{n}-S z_{n}\right\|=0 \text {, }
$$

Which in turn means that $\lim _{n \rightarrow \infty}\left\|x_{n}-S x_{n}\right\|=0$. By the definition of $S$ and $x_{n} \rightarrow u$, we have $S u=u$. This implies that $u \in S_{F i x}$. Hence $u \in T_{F i x} \cap S_{F i x}$. Next we show that $u \in \operatorname{SGMEP}\left(F_{1}, \cdots, F_{N}\right)$.

Note that

$$
\begin{gathered}
F_{1}\left(y, u_{n}^{1}\right)+\left\langle\eta\left(u_{n}^{1}, y\right), A_{1} u_{n}^{1}\right\rangle \leq \frac{1}{r_{n}^{1}}\left\|y-u_{n}^{1}\right\| \cdot\left\|J u_{n}^{1}-J x_{n}\right\|, \\
F_{2}\left(y, u_{n}^{2}\right)+\left\langle\eta\left(u_{n}^{2}, y\right), A_{2} u_{n}^{2}\right\rangle \leq \frac{1}{r_{n}^{2}}\left\|y-u_{n}^{2}\right\| \cdot\left\|J u_{n}^{2}-J u_{n}^{1}\right\|, \\
\vdots \\
F_{N}\left(y, u_{n}^{N}\right)+\left\langle\eta\left(u_{n}^{N}, y\right), A_{N} u_{n}^{N}\right\rangle \leq \frac{1}{r_{n}^{N}}\left\|y-u_{n}^{N}\right\| \cdot\left\|J u_{n}^{N}-J u_{n}^{N-1}\right\| \cdot
\end{gathered}
$$

From the above inequalities and $J$ is uniformly norm-to-norm continuous on bounded set, we get

$$
\lim _{n \rightarrow \infty}\left\|J u_{n}^{1}-J X_{n}\right\|=\lim _{n \rightarrow \infty}\left\|J u_{n}^{2}-J u_{n}^{1}\right\|=\cdots=\lim _{n \rightarrow \infty}\left\|J u_{n}^{N}-J u_{n}^{N-1}\right\| .
$$

From the latter equation and $A$ is $\eta$-monotone mapping, we abtain that $F_{i}(y, u)+\left\langle\eta(u, y), A_{i} y\right\rangle \leq 0, \forall y \in C, 1 \leq i \leq N$.

Let $t_{i} \in(0,1](1 \leq i \leq N), y \in C$. Noting

$y_{t}^{i}=t_{i} y+\left(1-t_{i}\right) u \in C, 1 \leq i \leq N$. We have

$$
\begin{aligned}
& 0=F_{i}\left(y_{t}^{i}, y_{t}^{i}\right)+\left\langle\eta\left(y_{t}^{i}, y_{t}^{i}\right), A_{i} y_{t}^{i}\right\rangle \\
& \leq F_{i}\left(y_{t}^{i}, y\right)+\left\langle\eta\left(y, y_{t}^{i}\right), A_{i} y_{t}^{i}\right\rangle(1 \leq i \leq N) .
\end{aligned}
$$

Let $t \rightarrow 0$, then $y_{t}^{i}=u \in C, i=1,2, \cdots, N$. So we get

$$
F_{i}(u, y)+\left\langle\eta(y, u), A_{i} u\right\rangle \geq 0, \forall y \in C, 1 \leq i \leq N .
$$

Thus we obtain $u \in \operatorname{SGMEP}\left(F_{1}, \cdots, F_{N}\right)$. Finally, we show that $u=\prod_{\Gamma} x_{0}$.From Lemma 2.3 and $x_{n}=\prod_{C} x_{0}$, Noting $\left\langle J x_{0}-J x_{n}, x_{n}-z\right\rangle \geq 0, \forall z \in C_{n}$. Since $\Gamma \subset C_{n}$, we also have $\left\langle J X_{0}-J X_{n}, x_{n}-p\right\rangle \geq 0, \forall p \in \Gamma$. Letting $n \rightarrow \infty$,we obtain $\left\langle J x_{0}-J u, u-p\right\rangle \geq 0, \forall p \in \Gamma$. By again Lemma 2.3, we can conclude that $u=\prod_{\Gamma} x_{0}$. This completes the proof.

\section{REFERENCES}

[1] E. Blum and W. Oettli, "From optimization and variational inequalities to equilibrium problems", Math.Stud. 1994, vol 63, pp. 123-145

[2] K. Wattanawitoon and P. Kumam, "Modified Proximal point algorithms for finding a zero point of maximal monotone operators, generalized mixed equilibrium problems and variational inequalities", journal of inequalities and applications, doi:10.1186/1029-242x-2012-118

[3] S. S. Zhang, "Generalized mixed equilibrium problem in Banach space", Applied Mathematics and Mechanics, 2009, vol.30:, pp. 1105-1112.

[4] K. Wattanawitoon and P. Kumam, "The Modified Block Iterative Algorithms for Asymptotically Relatively Nonexpansive Mappings and the System of Generalized Mixed Equilibrium Problems", Hindawi Publishing Corporation Journal of Applied Mathematics. doi:10.1155/2012/395760.

[5] W. Takahashi, Nonlinear Functional Analysis, Fixed Point Theory and Its Applications Yokohama Publishers, Yokohama, Japan, 2000.

[6] W. Takahasi and K. Zembayashi, "Strong and weak convergence theorems for equilibrium problems and relatively nonexpansive mappings in Banach spaces”, Nonlinear Anal. 2009, vol. 70:, pp. 45-57

[7] S. Kamimura and W. Takahashi, "Strong convergence of a proximal-type algorithm in a Banach space”, SIAM J. Optim. 2002, vol. 13 (3):, pp. 938-945

[8] Y. I. Alber, Metric and generalized projection operators in Banch space: properties and applications. In: Kartosator, A. G. eds. (1996) Theory and Ap pli cations of Nonlinear operators of Accretive and Monotone Type. Marcel Dekker, New York, pp. 15-50 